\title{
Changes in chemical coding of myenteric neurones in ulcerative colitis
}

\author{
M Neunlist, P Aubert, C Toquet, T Oreshkova, J Barouk, P A Lehur, M Schemann, \\ J P Galmiche
}

See end of article for authors' affiliations

......................

Correspondence to: Dr J P Galmiche,

Department of

Gastroenterology and

Surgery, Hôtel Dieu

Hospital, Place Alexis

Ricordeau, 44035 Nantes,

France;

galmiche@easynet.fr

or

Dr M Neunlist, INSERM U 539, Place Alexis

Ricordeau, 44035 Nantes,

France; michel.neunlisł@

sante.univ-nantes.fr

Accepted for publication 30 July 2002

\begin{abstract}
Background: Morphological and functional changes in the enteric nervous system (ENS) have been reported in inflammatory bowel diseases but it is still uncertain whether neurochemical coding of myenteric neurones is altered in ulcerative colitis (UC).

Aims: In this study we investigated transmitter co-localisation in myenteric neurones of normal colon and the colon of patients with UC.

Methods: Choline acetyltransferase (ChAT), neurone specific enolase (NSE), vasoactive intestinal peptide (VIP), and substance P (SP) were detected by immunohistochemical methods in whole mounts of colonic myenteric plexus of UC patients $(n=10)$ and controls $(n=8)$.

Results: The proportion of ChAT positive and VIP positive neurones relative to the NSE population did not differ in inflamed (33.3\% and $9.3 \%$, respectively) and non-inflamed segments $(33.6 \%$ and $9.7 \%)$ of UC colon compared with controls $(35.0 \%$ and $6.9 \%)$. The proportion of SP positive neurones was significantly larger in both inflamed $(15.5 \%)$ and non-inflamed $(20.3 \%)$ segments than in controls $(5.9 \%)$. Analysis of changes in subpopulations showed that $26.9 \%$ of neurones were only ChAT positive in controls but that the proportion was significantly smaller in inflamed (18.8\%) and non-inflamed $(15.8 \%)$ areas of UC. The proportions of neurones containing ChAT and SP were significantly higher in inflamed (11.8\%) and non-inflamed (13.9\%) areas than in controls $(5.0 \%)$.

Conclusion: Remodelling of myenteric neurones in UC involves a shift from mainly cholinergic to more SP positive innervation. This effect may constitute part of the neuronal basis for the motility disturbances observed in UC.
\end{abstract}

$\mathrm{T}$ wo major forms of inflammatory bowel disease (IBD), Crohn's disease (CD) and ulcerative colitis (UC), can be distinguished by their clinical manifestations, inflammation site in the gut, immunological profile, and probably their aetiology. ${ }^{1}$ Moreover, changes in the enteric nervous system (ENS) have been observed during IBD, encompassing modifications in morphology (that is, neuronal hyperplasia and large internodal fibre tracts), neuromediator content of enteric neurones, and changes in the function of enteric neurones (impaired release of neuromediators) (for a review, see Geboes and Collins ${ }^{2}$ ). These changes in enteric neurones are likely to play a role in the altered motility and secretory responses observed in IBD.

Previous studies reported changes in gut neuromediators of patients with UC and CD. These studies were mainly concerned with characterising the distribution of nerves and their peptide content and determining the concentrations of various peptides within the gut of $\mathrm{CD}$ or UC patients. Alterations in the populations of vasoactive intestinal peptide (VIP) and substance P (SP) were mainly observed, but the results obtained were often contradictory for the same pathology. UC studies reported increased SP levels in the mucosa or whole tissue, ${ }^{3-6}$ a decrease in SP immunoreactive nerves, ${ }^{7}$ or even no change in SP levels in colonic mucosa compared with controls. ${ }^{8}$ Concerning changes in VIP, no clear trend has been identified. Surrenti and colleagues ${ }^{8}$ showed a decrease in mucosal VIP level while Tomita and colleagues ${ }^{9}$ found an increase of VIPergic innervation patterns in UC and control patients. O'Morain and colleague ${ }^{10}$ observed an increase in VIP in UC while no alterations in VIP or SP content were reported by Koch and colleagues. ${ }^{3}$

One reason for these apparent discrepancies may be differences in drug treatment, duration or inflammatory degree of the disease, or the region of the gut studied. Differences could also result in part from the difficulty in obtaining representative or quantitative measurements when performing studies on thin tissue sections. In addition, whether changes in peptide content within fibres innervating the gut during UC are related to changes in neuromediator content within the enteric plexus is not known at present. Therefore, a complementary approach to studies aimed at characterising changes in fibre content in UC is to determine the putative changes in neuronal content within the enteric plexus using immunohistochemical methods on whole mounts of the myenteric or submucosal plexus in which the integrity of the network is preserved. This method has been successfully applied to characterise changes in the myenteric and submucosal plexus in $\mathrm{CD} .{ }^{11}{ }^{12}$ Moreover, this method, by determining co-localisation of the different neuromediators within a single neurone, allows the neurochemical code of the affected population to be established. This is of pivotal importance as neurones with different coexisting neurochemical markers probably have different functions. ${ }^{13}$ Therefore, identification of changes in the neurochemical code of these populations could explain in part the changes in gastrointestinal functions observed in these pathologies.

There is a lack of information on the putative changes in cholinergic, SP immunoreactive, and VIP immunoreactive populations of myenteric neurones in patients with UC. As no data are available for the human colon, our investigations were

Abbreviations: $C D$, Crohn's disease; ChAT, choline acetyltransferase ENS, enteric nervous system; IBD, inflammatory bowel disease; NSE, neurone specific enolase; SP, substance P; VIP, vasoactive intestinal peptide; UC, ulcerative colitis; PBS, phosphate buffered saline; NK-1, neurokinin 1 . 


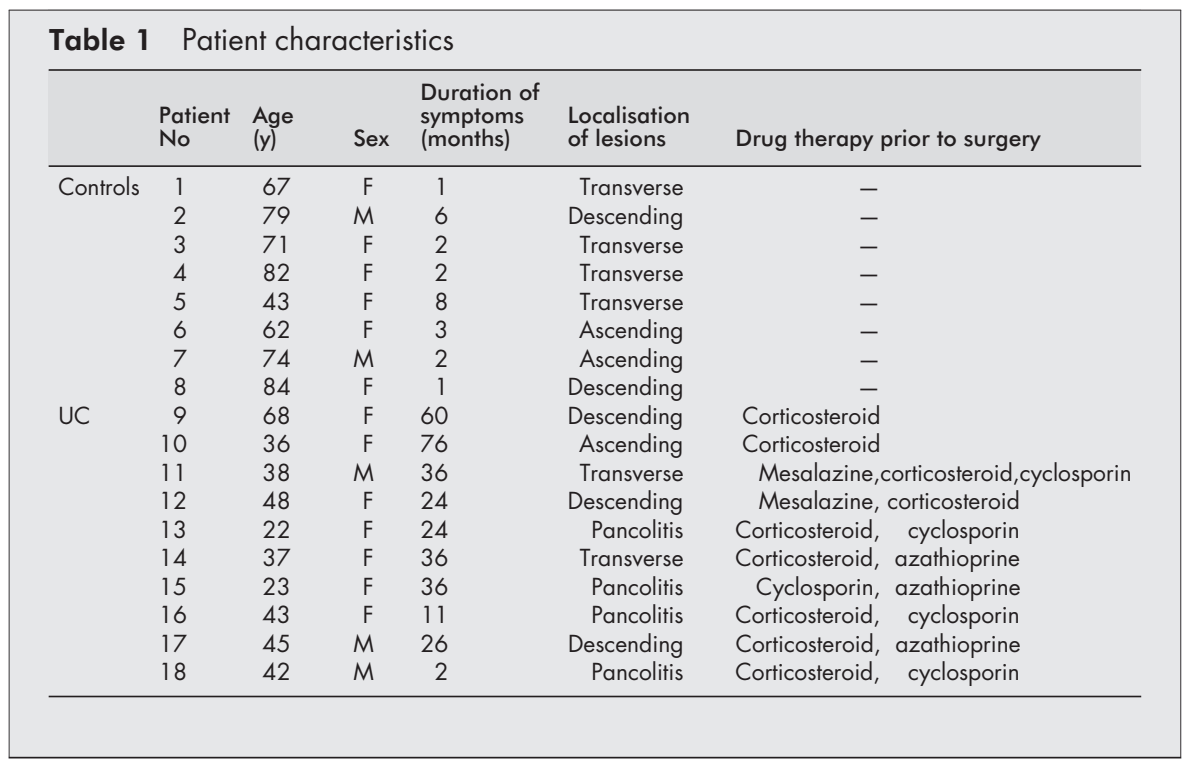

Table 2 Classification of lesions in tissue samples of ulcerative colitis (non-inflamed versus inflamed), according to Geboes and colleagues ${ }^{14}$

\begin{tabular}{llllllll}
\hline & \multicolumn{7}{c}{ Geboes classification scores* } \\
\cline { 2 - 8 } & 0 & 1 & 2 & 3 & 4 & 5 \\
\hline Non-inflamed segments $(n=6)$ & 2 & 3 & 1 & 0 & 0 & 0 \\
Inflamed segments $(n=9)$ & 0 & 0 & 0 & 1 & 0 & 8 \\
\hline
\end{tabular}

*Grades are: 0, structural changes only; 1, chronic inflammation; 2, lamina propria neutrophils; 3 ,

neutrophils in epithelium; 4 , crypt destruction; 5 , erosions or ulcers.

directed first at determining the patterns of transmitter choline acetyltransferase (ChAT), VIP, and SP co-localisation in the myenteric plexus of the healthy human colon. Changes in neurochemically distinct populations were then investigated in the colon of patients with UC, and inflamed and non(or mildly) inflamed segments were compared.

\section{MATERIALS AND METHODS}

\section{Specimens of normal colon}

Control tissue was obtained from eight patients (mean age 70.3 (4.7) years) undergoing surgical resection for colonic adenocarcinoma distant from the tumour (table 1). None of the patients had bowel obstruction or other colon disease.

\section{Specimens of colon with ulcerative colitis}

Tissue samples were taken from 10 patients (mean age 40.0 (4.1) years) undergoing proctocolectomy for active UC, whose disease had been diagnosed 2.8 (1.2) years before (see table 1 for clinical data). All patients, except one, were receiving steroid treatment, and six were on immunosuppressive drugs. Macroscopically inflamed segments and non- (or mildly) inflamed segments of colon were removed, except in three patients with pancolitis (patient Nos 10, 16, 18; table 1). Tissues from all patients were assessed histologically using the grading scale developed by Geboes and colleagues. ${ }^{14}$ Inflammation scores for the tissues are reported in table 2. Most of the macroscopically least inflamed regions had a score of 2 (subsequently referred to as "non-inflamed areas"). Most of the macroscopically inflamed regions had a score of 5 (subsequently referred to as "inflamed areas"). According to this scale, tissues from control patients all had a score of 0 .

\section{Tissue preparation}

Specimens of normal and diseased colon were obtained from patients undergoing surgery and immediately processed in the pathology department. The tissue was placed in $4^{\circ} \mathrm{C}$ oxygenated sterile Krebs solution containing (in $\mathrm{mM}$ ): $117 \mathrm{NaCl}, 4.7$ $\mathrm{KCl}, 1.2 \mathrm{MgCl}_{2} 6 \mathrm{H}_{2} \mathrm{O}, 1.2 \mathrm{NaH}_{2} \mathrm{PO}_{4}, 25 \mathrm{NaHCO}_{3}, 2.5 \mathrm{CaCl}_{2} 2 \mathrm{H}_{2} \mathrm{O}$, and 11 glucose. It was then rapidly transported to the laboratory for experiments.

The piece was pinned flat mucosa down in a dissection dish containing ice cold sterile oxygenated Krebs solution that was changed every 10-15 minutes. Preparations taken from intertaenial regions were $4-7 \mathrm{~cm}$ in the circumferential direction and $4-5 \mathrm{~cm}$ in the longitudinal direction. The tissue was washed 4-6 times with sterile Krebs solution and pinned back in a sterile Sylgard coated Petri dish before addition of sterile culture medium $(40 \mathrm{ml})$. The culture medium (Dulbecco's modified Eagle's medium/F12; Sigma, St Louis, Missouri, USA) was supplemented with $10 \%$ heat inactivated fetal calf serum (Sigma), $100 \mathrm{IU} / \mathrm{ml}$ penicillin, $100 \mu \mathrm{g} / \mathrm{ml}$ streptomycin, 1.1 $\mu \mathrm{g} / \mathrm{ml}$ amphotericin B, $20 \mu \mathrm{g} / \mathrm{ml}$ gentamicin (Sigma), glutamine, and $2.1 \mathrm{~g} / \mathrm{l} \mathrm{NaHCO}$. To enhance immunoreactivity for peptides in nerve cell bodies, colchicine $(40 \mu \mathrm{M})$ was added to the medium. The tissue was then maintained at $37^{\circ} \mathrm{C}$ overnight in a humidified incubator containing $5 \% \mathrm{CO}_{2}$ and air, and continuously agitated on a rocking tray.

\section{Immunohistochemistry}

After culture, the tissue was fixed for 4-6 hours in $0.1 \mathrm{M}$ phosphate buffered saline (PBS) containing 4\% paraformaldehyde at room temperature. Following several washes in PBS, the tissue was pinned in a dissection dish. Under a dissection microscope, the circular muscle was removed to expose the myenteric plexus. Thin fibres and connective tissue of the 


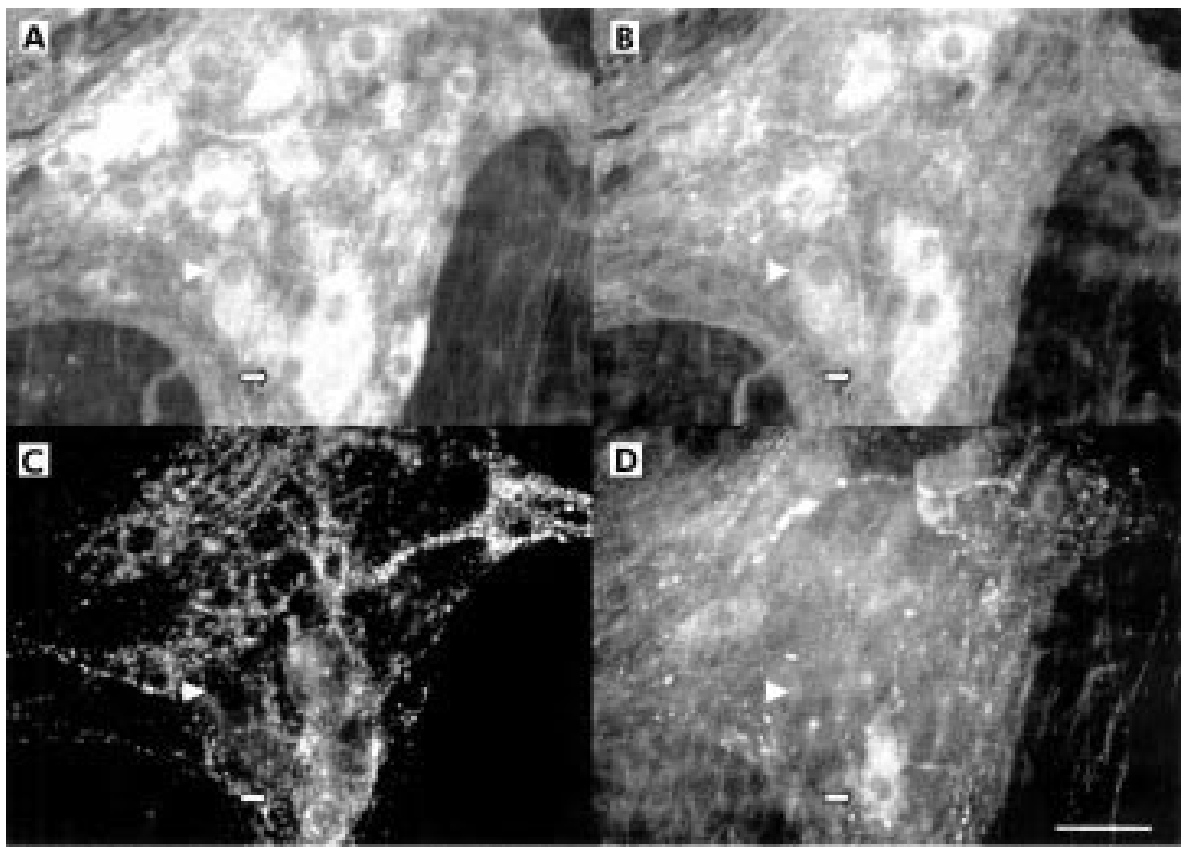

Figure 1 Immunohistochemical detection of transmitter coding of human myenteric plexus from control patients. Quadruple labelling with antibodies against neurone specific enolase (NSE), choline acetyltransferase (ChAT), substance P (SP), and vasoactive intestinal peptide (VIP) performed in a myenteric ganglion showed various subpopulations. A ganglion was labelled with NSE to identify myenteric neurones (A) ChAT neurones (B) formed the largest population identified and were not usually co-localised with SP or VIP (arrowhead). No SP positive neurones (C) and few VIP positive neurones (D) were identified in this ganglion. VIP positive neurones were not co-localised with ChAT or SP (filled arrow). Scale bar: $25 \mu \mathrm{m}$.

serosa were also removed. The myenteric plexus/longitudinal muscle was then permeabilised for $1-2$ hours in $\mathrm{PBS} / \mathrm{NaN}_{3}$ containing $0.5 \%$ Triton X 100 and $4 \%$ horse serum.

The tissue was incubated with the following primary antibodies diluted in $\mathrm{PBS} / \mathrm{NaN}_{3}, 4 \%$ horse serum, and $0.5 \%$ Triton $X$ for 18-20 hours at room temperature: rabbit anti-ChAT $\left(1: 2000^{17}\right)$, rat anti-substance P (1:1000; Fitzgerald, Concord, USA), and mouse anti-VIP (1:1000; Biogenesis, UK). Following incubation with primary antisera, the tissue was washed with PBS and incubated for 2-3 hours with donkey antirabbit IgG conjugated to carboxymethylindocyanine (1:500), donkey antirat IgG conjugated to 7-amino-4indodicarbocyanin (1:500), and donkey antimouse IgG conjugated to 7-amino-4-methyl-coumarin-3-acetate (1:50) (from Jackson Labs, purchased from Euromedex, France). In the following step, the tissue was labelled with rabbit anti-NSE (1:3000; Polysciences, Eppelheim, Germany) for 18-20 hours. Following incubation with primary antisera, the tissue was washed with PBS and incubated for 2-3 hours with donkey antirabbit IgG conjugated to fluorescein isothiocyanate (1:200).

The specificity of VIP antiserum was checked because preabsorption of mouse anti-VIP with $10 \mu \mathrm{M}$ of human VIP resulted in complete loss of tissue immunoreactivity. The specificity of ChAT antiserum and monoclonal SP antibody had been assessed previously. ${ }^{15}$

Specimens were viewed under an Olympus IX 50 fluorescence microscope fitted with adequate filter cubes. Pictures were acquired with a black and white video camera (Mod. 4910; Cohu Inc., SL Microtest, Jena, Germany) connected to a Macintosh computer via a frame grabber card (Scion Image, SL Microtest).

\section{Identification of the neuronal cell population}

To determine the general coding of the myenteric plexus, a total of 20 myenteric ganglia were analysed in each preparation. An anti-NSE antibody was used as a general neuronal marker to determine the total number of cell bodies in the myenteric plexus. To determine the proportion of ChAT, VIP, and SP immunoreactive populations in myenteric neurones, quadruple immunohistochemistry was performed on the same preparation. The proportions of neurochemically identified populations are expressed relative to the number of NSE positive neurones per ganglion, which represents $100 \%$ of neurones. For example, the code "ChAT/- neurones" is attributed to those immunoreactive for ChAT alone, and "ChAT/SP/- neurones" to those immunoreactive for ChAT and SP, but not VIP.

\section{Statistics}

Data are expressed as mean (SEM) when normally distributed or as median (25-75\% percentiles) when non-normally distributed. A paired or unpaired $t$ test and one way analysis of variance (ANOVA) followed by a Bonferroni $t$ test were performed to compare different populations. ANOVA on Ranks followed by a Dunn's test was performed when data were not normally distributed. Correlations were performed using the Pearson product moment correlation. Differences were considered significant when $\mathrm{p}<0.05$.

\section{RESULTS}

\section{General coding in human colonic myenteric plexus}

Whole mounts of myenteric plexus were obtained and labelled simultaneously with antibodies against NSE, ChAT, VIP, and SP. This allowed characterisation of the precise neurochemical coding of myenteric neurones in control tissue (fig l), as well as in tissues from non-inflamed (fig 2) and inflamed (fig 3) areas.

The average number of NSE positive neurones per ganglia was similar in control patients $(2 \mathrm{l}(3) ; \mathrm{n}=8)$ and in non-inflamed and inflamed areas in UC ( $17(3)(\mathrm{n}=6) v 21(2)$ $(n=9)$, respectively).

The average number of ChAT positive neurones was 35.0 (3.0)\% of NSE positive neurones in control tissue $(n=8)$. This proportion was similar to that observed in both non-inflamed and inflamed areas in UC (table 3). VIP positive neurones were found in equal proportions in control samples and in those from 


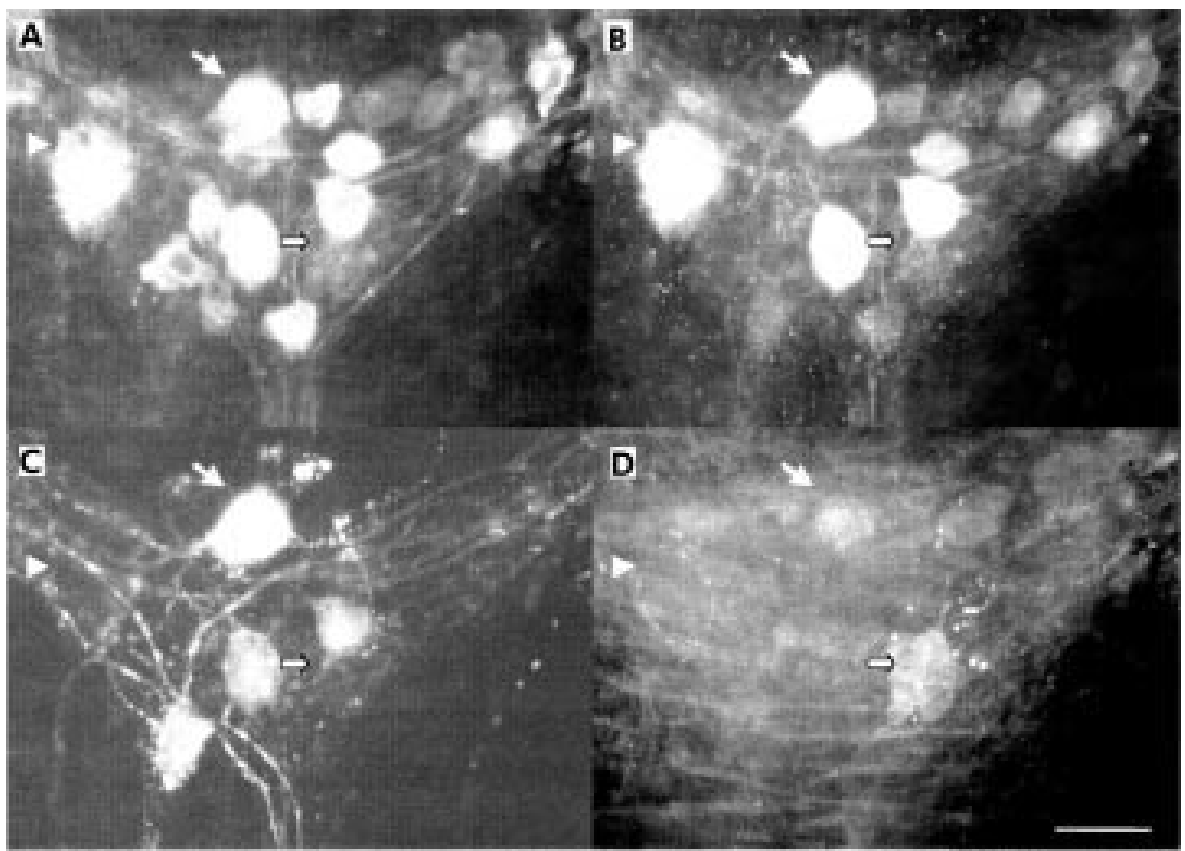

Figure 2 Immunohistochemical detection of transmitters in the myenteric plexus of patients with ulcerative colitis in non-inflamed areas. Quadruple immunohistochemistry for neurone specific enolase (NSE) (A), choline acetyltransferase (ChAT) (B), substance P (SP) (C), and vasoactive intestinal peptide (VIP) (D) revealed that a similar proportion of ChAT positive neurones were immunorective for SP (arrow) or did not contain either SP or VIP (arrowhead). In addition, VIP positive neurones were not co-localised with ChAT or SP (filled arrow). Scale bar: 25 $\mu \mathrm{m}$.

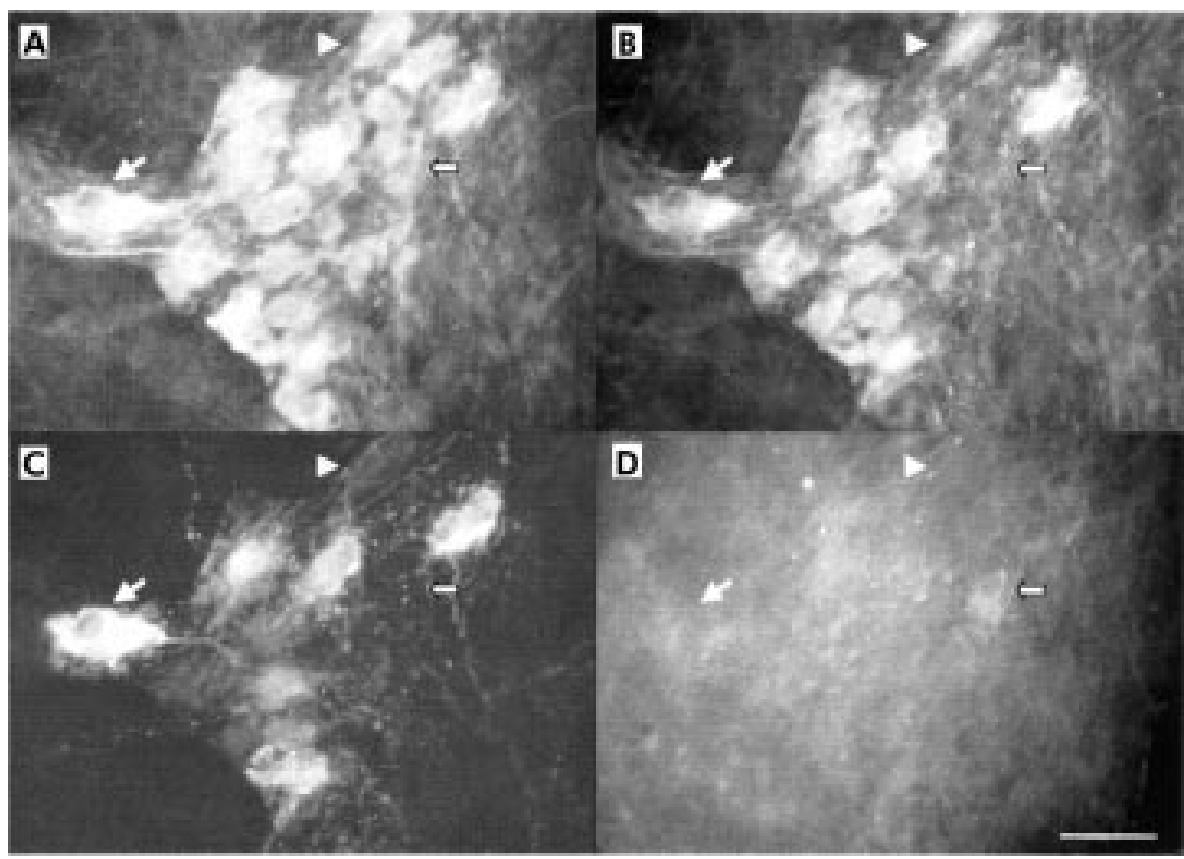

Figure 3 Immunohistochemical detection of transmitters in the myenteric plexus of patients with ulcerative colitis in inflamed areas. Quadruple immunohistochemistry for neurone specific enolase (NSE) (A), choline acetyltransferase (ChAT) (B), substance P (SP) (C), and vasoactive intestinal peptide (VIP) (D) revealed that a large proportion of ChAT positive neurones were immunorective for SP (arrow). Some ChAT positive neurones did not contain either SP or VIP (arrowhead). In addition, VIP positive neurones were not co-localised with ChAT or SP (filled arrow). Scale bar: $25 \mu \mathrm{m}$.

non-inflamed and inflamed areas (table 3). SP immunoreactive neurones formed $5.9(0.7) \%$ of NSE positive neurones in controls. In UC, there was a significant fourfold increase in the proportion of SP positive neurones in non-inflamed areas $(\mathrm{n}=6$; $\mathrm{p}<0.0001)$ and a significant threefold increase in inflamed areas $(\mathrm{n}=9 ; \mathrm{p}<0.0001)$ compared with controls (table 3$)$.

For controls and UC patients, no significant correlation was found between the age of patients and the neurochemical code for ChAT, SP, or VIP populations. Likewise, no correlation was found between disease duration, inflammatory grade, and the proportion of the population immunoreactive for ChAT, SP, or VIP.

Subpopulations of neurochemically identified neurones The different neurochemically identified populations are described in table 4. Based on their neurochemical coding, five 
Table 3 Proportion of choline acetyltransferase (ChAT), vasoactive intestinal peptide (VIP), and substance $P(S P)$ in myenteric neurones from controls and patients with ulcerative colitis in inflamed and non-inflamed areas

\begin{tabular}{llcc}
\hline & Control & Non-inflamed & Inflamed \\
\hline ChAT & $35.0(3.0)$ & $33.6(2.6)$ & $33.3(2.3)$ \\
VIP & $6.9(1.4)$ & $9.7(3.8)$ & $9.3(3.1)$ \\
SP & $5.9(0.7)$ & $20.3(2.8)^{* * *}$ & $15.5(1.1)^{* * *}$ \\
\hline
\end{tabular}

Values are mean (SEM)

$* * * p<0.0001$ compared with controls.

distinct neuronal populations were identified in controls. The population of ChAT/- neurones formed the significantly largest cholinergic subpopulation (27\% of NSE labelled neurones). ChAT/SP/-, ChAT/VIP/-, VIP/-, and SP/- subpopulations had equal proportions. No myenteric neurones immunoreactive for ChAT/SP/VIP or SP/VIP were present in control tissue.

Seven distinct subpopulations were identified in UC non-inflamed areas. ChAT/- neurones and ChAT/SP/- neurones formed the largest subpopulations, showing similar proportions. ChAT/VIP/-, VIP/-, and SP/- subpopulations had identical proportions. Very few neurones were immunoreactive for ChAT/VIP/SP and VIP/SP/-.

In inflamed areas of UC segments, ChAT/- and ChAT/SP/neurones formed the largest subpopulations, showing equal proportions. ChAT/VIP/-, VIP/-, and SP/- subpopulations formed equally small proportions of neurones. ChAT/SP/VIP and SP/VIP/- subpopulations were also identified.

\section{Subpopulations exhibiting plasticity in UC}

Our study revealed significant changes in the proportion of three neurochemically identified subpopulations. Firstly, the proportion of the ChAT/- population decreased by $41 \%$ in non-inflamed areas and by $30 \%$ in inflamed areas compared with control tissue (table 4, fig 4). In addition, no differences were observed in this population between non-inflamed and inflamed areas. Secondly, the ChAT/SP/- population increased about twofold in non-inflamed and inflamed sites of UC compared with controls (table 4, fig 4). For this subpopulation, no difference was found in the proportions in non-inflamed and inflamed areas. Thirdly, the proportion of SP/- neurones was significantly increased in non-inflamed areas of UC: $4.2 \%$ ( (25-75th percentiles $1.8-7.2) ; \mathrm{p}<0.01 ; \mathrm{n}=6)$ and $2.4 \%((25-$ 75th percentiles $1.3-4.3) ; \mathrm{p}<0.05 ; \mathrm{n}=9$ ) in inflamed areas compared with controls $(0.4 \%$ (25-75th percentiles $0.1-0.9)$; $\mathrm{n}=8)$.

\section{DISCUSSION}

This study, performed on whole mounts of human myenteric plexus, identified the transmitter co-localisation pattern in neurones of control and UC patients. In UC, alterations occurred in the proportion of ChAT/-, ChAT/SP/-, and SP/populations compared with control tissue. These changes were of a similar proportion in inflamed and non-inflamed areas in UC.

Use of a combination of antibodies against ChAT, VIP, and SP allowed us to characterise transmitter co-localisation in colonic myenteric neurones, showing five distinct subpopulations (ChAT/-, ChAT/SP, ChAT/VIP/-, VIP/-, and SP/-) and a proportion of non-immunoreactive neurones for the antigen studied. The largest neuronal population identified was ChAT immunoreactive (35\%) and hence likely to be cholinergic. This proportion was lower than that reported by Porter and colleagues ${ }^{16}$ who found that $64 \%$ of NSE positive myenteric neurones were ChAT immunoreactive in normal human colon.
The fact that these authors maintained tissue in organotypic culture for four days to enhance immunoreactivity may account for this discrepancy. The largest cholinergic subpopulation identified in the myenteric plexus was ChAT/-. VIP formed $9 \%$ of the total NSE population, a proportion similar to that reported in the human small intestine $\left(5 \%{ }^{17}\right)$. Most SP neurones were co-localised with ChAT, which was also the case in the submucosal plexus of the human rectum ${ }^{12}$ and the myenteric plexus of the guinea pig. ${ }^{13}$ Although ChAT and VIP label the vast majority of neurones in the human submucosal plexus, ${ }^{12}{ }^{18}$ this is not true for the human myenteric plexus (present study) or the guinea pig myenteric plexus. ${ }^{13}$

In UC, there was a threefold increase in the proportion of myenteric neurones immunoreactive for SP whereas the proportion of ChAT and VIP positive neurones was not affected. Because of age differences between control patients and UC patients, one could attribute the alterations observed in part to an age related phenomena. This is however unlikely as in control patients no correlation between age and the proportion of SP positive neurones was observed. Although this observation must be viewed with caution due to the relatively low number of patients, it is further supported by the work of Johnson and colleagues ${ }^{19}$ who also found no changes with age in both ChAT and VIP populations. Moreover, in a study performed in control and UC patients of the same age, a significant increase in SP content was observed in UC. ${ }^{20}$ In contrast, in CD patients of a similar age to UC patients, no changes in the SP population were observed. These arguments strongly suggest that the increase in SP observed in this study and by others is a hallmark of UC. In addition, as the majority of UC patients were under corticosteroid treatment, we cannot exclude the fact that this treatment induced neuronal plasticity. Nevertheless, this also seems unlikely as a recent study characterising expression of the SP receptor and mRNA found no correlation in corticosteroid treated and naive UC patients. ${ }^{21} 22$

Previous studies have reported an increase in the density of SP immunoreactive fibres and SP content in UC. This increase was mainly observed in the lamina propria. ${ }^{5620}$ Increasing the SP level in myenteric neurones projecting to the mucosa could in part explain this observation. In the guinea pig, a proportion of myenteric ChAT/SP and ChAT positive neurones projected to the mucosa ${ }^{23} 24$ and functioned as intrinsic sensory neurones responding to stretch and various chemical stimuli. ${ }^{25}{ }^{26}$ Accordingly, myenteric neurones projecting to the mucosa were identified in human ileum. ${ }^{27}$

Future studies should address the questions of whether the changes in SP observed in myenteric neurones are the result of transductional, post-transductional, or even alterations in peptide transport. Indeed, although alterations in the transport of peptides along fibres could explain the increase in SP in myenteric neurones in UC, it seems unlikely. Indeed, differences in SP between UC and control patients were observed after incubation of the tissue for 16-18 hours in colchicine which blocks axonal transport of peptides. Changes in mRNA levels for SP receptors were already observed in $\mathrm{UC}^{22}$ but it is not known whether SP mRNA is increased in myenteric neurones or whether degradation of SP by the degradation compartments is altered in UC.

The changes observed in our study for UC differ from those for $\mathrm{CD}$. The proportion of VIP positive neurones in CD increased (compared with controls) in inflamed areas of the myenteric plexus ${ }^{11}$ and at sites distant from inflammation in the submucosal plexus. ${ }^{12}$ Moreover, no changes were observed in $\mathrm{CD}$ in the population of submucosal neurones immunoreactive for $\mathrm{SP}^{12}$ and in nerve fibres immunoreactive for $\mathrm{SP}^{3}$

The increase in SP positive neurones observed in UC occurred primarily in the population of ChAT/- neurones detected in control patients. In fact, the total proportion of ChAT positive neurones was not modified in UC compared with controls, and the increase in the proportion of ChAT/SP/neurones was equivalent to the decrease in the proportion of 
Table 4 Neurochemical code of colonic myenteric neurones in control tissues and tissues from non-inflamed and inflamed areas in ulcerative colitis

\begin{tabular}{lccc}
\hline & Control & Non-inflamed & Inflamed \\
\hline ChAT/- & $26.9(2.7)$ & $15.8(2.8)^{* *}$ & $18.8(2.4)^{*}$ \\
ChAT/SP/- & $5.0(0.5)$ & $13.9(1.7)^{* *}$ & $11.8(1.4)^{* *}$ \\
ChAT/VIP/- & $2.6(0.7)$ & $3.7(2.1)$ & $2.4(0.7)$ \\
ChAT/VIP/SP/- & $0.4(0.1)$ & $1.4(0.7)$ & $0.7(0.2)$ \\
VIP/SP/- & $0.0(0.0)$ & $0.2(0.1)$ & $0.1(0.1)$ \\
VIP/- & $4.0(1.0)$ & $4.5(1.9)$ & $6.2(2.7)$ \\
SP/- & $0.6(0.2)$ & $5.0(1.7)$ & $3.1(0.7)^{*}$ \\
\hline Values are mean (SEM). & & \\
*p<0.05, ** $p<0.01$ compared with controls. & \\
ChAT, choline acetyltransferase; SP, substance P; VIP, vasoactive intestinal peptide.
\end{tabular}

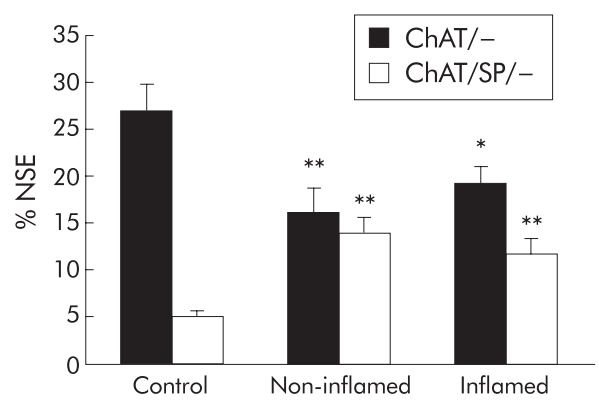

Figure 4 Histogram of the proportion of ChAT/- and ChAT/SP/myenteric neurones from control and ulcerative colitis patients in non-inflamed and inflamed areas. ${ }^{*} p<0.05,{ }^{* *} p<0.01$ compared with controls. Values are mean (SEM). ChAT, choline acetyltransferase; SP, substance P; NSE, neurone specific enolase.

the ChAT/- population observed in UC. Neuronal tracing methods have shown that a proportion of ascending myenteric neurones innervating colonic circular muscle are ChAT/- and ChAT/SP/- positive and could function as motoneurones. ${ }^{28}$ Therefore, it is tempting to speculate that at least some of these neurones are excitatory muscle motoneurones. In fact, acetylcholine and SP stimulated small bowel motility in healthy volunteers. ${ }^{29}{ }^{30} \mathrm{ChAT} /-$ and ChAT/SP/- neurones could also be intrinsic primary afferent neurones projecting to the mucosa. ${ }^{25}$

What might be the clinical relevance of the SP increase in UC? Firstly, SP may play an important role in the pathophysiology of IBD. ${ }^{31}$ There is increased expression of SP binding sites in UC and $\mathrm{CD}^{21}$ as well as an increase in neurokinin 1 (NK-1) receptor mRNA. ${ }^{22}$ NK-1 receptors are found on Cajal cells where they could be involved in motility regulation, ${ }^{32}$ and on blood vessels and lymphoid structures. ${ }^{33}$ In an animal model of colitis induced by dextran sulphate, NK-1 antagonist reduced colonic inflammation. ${ }^{34}$ In the same animal model, inflammation induced an increase in SP synthesis in myenteric neurones. ${ }^{35}$ Moreover, a decrease in neutral endopeptidase (EC 3.4.24.11) activity (which degrades SP) was observed in Trichinella infected intestine in conjunction with increased SP levels. ${ }^{36}$ These combined effects-that is, increased SP level and downregulation of endopeptidase activity-could result in a net increase in SP that might contribute to uncontrolled inflammation.

A surprising finding was that alterations in the neurochemical code of myenteric neurones occurred in similar proportions in inflamed and non-inflamed areas. However, this result is consistent with data obtained in an animal model of experimental colitis showing similar functional alterations in sympathetic innervation at inflamed and non-inflamed sites.$^{37}$ Furthermore, altered motility was observed at a remote non-inflamed site and at inflamed sites in UC patients or animal models of IBD. ${ }^{38-40}$ In UC patients, fasting motility was increased compared with controls. ${ }^{41}$ This could have been due in part to an SP increase in myenteric neurones, as it has been shown that inflammation of the colon of conscious dogs enhanced SP stimulation of giant migrating contractions compared with controls. ${ }^{42}$ SP induction during UC in non-inflamed and inflamed areas could be due to an increase in inflammatory cytokines such as interleukin $1 \beta$, which have been observed during IBD in the intestine. ${ }^{1}$ In fact, interleukin $1 \beta$ induced increased SP expression in rat myenteric fibres. ${ }^{43}$

In summary, our study showed that marked changes in the neurochemical coding of myenteric neurones occur during UC. ChAT/- neurones were identified as the putative neuronal population exhibiting neural plasticity by expressing SP. Therefore, this remodelling in UC occurs as a shift from mainly cholinergic to more peptidergic innervation. Similar changes in neurochemical coding were also observed in the least affected sites. This effect may constitute part of the neuronal basis for the altered motility disturbance observed during UC.

\section{ACKNOWLEDGEMENTS}

This work was supported in part by grants from the IRMAD (Institut de Recherche des Maladies de l'Appareil Digestif; Astra Zeneca, France) and from the Conseil Régional des Pays de Loire. The authors are grateful for the valuable assistance provided by the surgical teams and the Department of Pathology.

\section{Authors' affiliations}

M Neunlist, P Aubert, T Oreshkova, INSERM U 539, Place Alexis Ricordeau, Nantes, France

C Toquet, Department of Pathology, University Hospital Hôtel Dieu, Nantes, France

J Barouk, J P Galmiche, INSERM U 539, Place Alexis Ricordeau, and Department of Gastroenterology and Surgery, University Hospital Hôtel Dieu, Nantes, France

P A Lehur, Department of Gastroenterology and Surgery, University Hospital Hôtel Dieu, Nantes, France

M Schemann, School of Veterinary Medicine, Department of Physiology, Hannover, Germany

\section{REFERENCES}

1 Fiocchi C. Inflammatory bowel disease: etiology and pathogenesis. Gastroenterology 1998;115:182-205.

2 Geboes K, Collins S. Structural abnormalities of the nervous system in Crohn's disease and ulcerative colitis. Neurogastroenterol Motil 1998; 10:189-202.

3 Koch TR, Carney JA, Go VL. Distribution and quantitation of gut neuropeptides in normal intestine and inflammatory bowel diseases. Dig Dis Sci 1987; 32:369-76.

4 Watanabe T, Kubota Y, Muto T. Substance P containing nerve fibers in ulcerative colitis. Int J Colorectal Dis 1998;13:61-7.

5 Keranen $U$, Kiviluoto $T$, Jarvinen $H$, et al. Changes in substance P-immunoreactive innervation of human colon associated with ulcerative colitis. Dig Dis Sci 1995;40:2250-58.

6 Vento P, Kiviluoto T, Keranen U, et al. Quantitative comparison of growth-associated protein- 43 and substance $P$ in ulcerative colitis. J Histochem Cytochem 2001;49:749-58.

7 Renzi D, Mantellini P, Calabro A, et al. Substance P and vasoactive intestinal polypeptide but not calcitonin gene-related peptide 
concentrations are reduced in patients with moderate and severe ulcerative colitis. Ital J Gastroenterol Hepatol 1998;30:62-70.

8 Surrenti C, Renzi D, Garcea MR, et al. Colonic vasoactive intestinal polypeptide in ulcerative colitis. J Physiol (Paris) 1993;87:307-11.

9 Tomita R, Tanjoh K, Fujisaki S, et al. Peptidergic nerves in the colon of patients with ulcerative colitis. Hepatogastroenterology 2000;47:400-4.

10 O'Morain C, Bishop AE, McGregor GP, et al. Vasoactive intestinal peptide concentrations and immunocytochemical studies in rectal biopsies from patients with inflammatory bowel disease. Gut 1984:25:57-61.

11 Belai A, Boulos PB, Robson T, et al. Neurochemical coding in the small intestine of patients with Crohn's disease. Gut 1997;40:767-74.

12 Schneider J, Jehle E, Starlinger M, et al. Neurotransmitter coding of enteric neurones in the submucous plexus is changed in non-inflamed rectum of patients with Crohn's disease. Neurogastroenterol Motil 2001;13:255-64.

13 Costa M, Brookes SJ, Steele PA, et al. Neurochemical classification of myenteric neurons in the guinea-pig ileum. Neuroscience 1996; 75:949-67.

14 Geboes K, Riddell R, Ost A, et al. A reproducible grading scale for histological assessment of inflammation in ulcerative colitis. Gut 2000;47:404-9

15 Schemann M, Sann H, Schaaf C, et al. Identification of cholinergic neurons in enteric nervous system by antibodies against choline acetyltransferase. Am J Physiol 1993;265:G1005-9.

16 Porter AJ, Wattchow DA, Brookes SJ, et al. Choline acetyltransferase immunoreactivity in the human small and large intestine. Gastroenterology 1996;111:401-8.

17 Dhatt N, Buchan AM. Colocalization of neuropeptides with calbindin D28k and NADPH diaphorase in the enteric nerve plexuses of normal human ileum. Gastroenterology 1994;107:680-90

18 Aubé AC, Lehur PA, Leborgne J, et al. Characterisation of submucosal circuits innervating the mucosa of the human sigmoid colon. Gut 2000;47(suppl III):A212.

19 Johnson RJ, Schemann M, Santer RM, et al. The effects of age on the overall population and on sub-populations of myenteric neurons in the rat small intestine J Anat 1998;192:479-88.

20 Goldin E, Karmeli F, Selinger Z, et al. Colonic substance P levels are increased in ulcerative colitis and decreased in chronic severe constipation. Dig Dis Sci 1989;34:754-7.

21 Mantyh CR, Vigna SR, Bollinger RR, et al. Differential expression of substance $P$ receptors in patients with Crohn's disease and ulcerative colitis. Gastroenterology 1995; 109:850-60.

22 Goode T, O'Connell J, Anton $\mathrm{P}$, et al. Neurokinin-1 receptor expression in inflammatory bowel disease: molecular quantitation and localisation. Gut 2000;47:387-96.

23 Song ZM, Brookes SJ, Costa M. Identification of myenteric neurons which project to the mucosa of the guinea-pig small intestine. Neurosci Lett 1991;129:294-8.

24 Neunlist $M$, Schemann M. Projections and neurochemical coding of myenteric neurons innervating the mucosa of the guinea pig proximal colon. Cell Tissue Res 1997;287: 1 19-25

25 Furness JB, Kunze WA, Bertrand PP, et al. Intrinsic primary afferent neurons of the intestine. Prog Neurobiol 1998;54:1-18.
26 Neunlist M, Dobreva G, Schemann M. Characteristics of mucosally projecting myenteric neurones in the guinea-pig proximal colon. J Physiol 1999;517:533-46

27 Hens J, Vanderwinden JM, De Laet MH, et al. Morphological and neurochemical identification of enteric neurones with mucosal projections in the human small intestine. J Neurochem $2001 ; 76: 464-71$.

28 Porter AJ, Wattchow DA, Brookes SJ, et al. The neurochemical coding and projections of circular muscle motor neurons in the human colon. Gastroenterology 1997;113:1916-23.

29 Burleigh DE. Motor responsiveness of proximal and distal human colonic muscle layers to acetylcholine, noradrenaline, and vasoactive intestinal peptide. Dig Dis Sci 1990;35:617-21.

30 Warner FJ, Liu L, Lubowski DZ, et al. Circular muscle contraction, messenger signalling and localization of binding sites for neurokinin $A$ in human sigmoid colon. Clin Exp Pharmacol Physiol 2000;27:928-33.

31 Holzer P. Implications of tachykinins and calcitonin gene-related peptide in inflammatory bowel disease. Digestion 1998;59:269-83.

32 Epperson A, Hatton WJ, Callaghan B, et al. Molecular markers expressed in cultured and freshly isolated interstitial cells of Cajal. Am J Physiol 2000;279:C529-39.

33 Goode T, O'Connell J, Sternini C, et al. Substance P (neurokinin-1) receptor is a marker of human mucosal but not peripheral mononuclear cells: molecular quantitation and localization. J Immunol 1998;161:2232-40

34 Stucchi AF, Shofer S, Leeman S, et al. NK-1 antagonist reduces colonic inflammation and oxidative stress in dextran sulfate-induced colitis in rats. Am J Physiol 2000;279:G1298-6.

35 Kishimoto S, Kobayashi H, Machino H, et al. High concentration of substance $\mathrm{P}$ as possible transmission of abdominal pain in rats with chemical induced ulcerative colitis. Biomed Res 1994;15/suppl 2): 133-40.

36 Hwang L, Leichter R, Okamoto A, et al. Downregulation of neutral endopeptidase (EC 3.4.24.11) in the inflamed rat intestine. Am J Physiol 1993;264:G735-43.

37 Jacobson K, McHugh K, Collins SM. Experimental colitis alters myenteric nerve function at inflamed and noninflamed sites in the rat. Gastroenterology 1995;109:718-22.

38 Manousos ON, Salem SN. Abnormal motility of the small intestine in ulcerative colitis. Gastroenterologia 1965;104:249-57.

39 Rao SS, Read NW, Brown C, et al. Studies on the mechanism of bowel disturbance in ulcerative colitis. Gastroenterology 1987;93:934-40.

40 Aube AC, Cherbut C, Barbier M, et al. Altered myoelectrical activity in noninflamed ileum of rats with colitis induced by trinitrobenzene sulphonic acid. Neurogastroenterol Motil 1999;11:55-62.

41 Coulie B, Camilleri M, Bharucha AE, et al. Colonic motility in chronic ulcerative proctosigmoiditis and the effects of nicotine on colonic motility in patients and healthy subjects. Aliment Pharmacol Ther 2001;15:653-63

42 Tsukamoto M, Sarna SK, Condon RE. A novel motility effect of tachykinins in normal and inflamed colon. Am J Physiol 1997;272:G1607-14.

43 Hurst SM, Stanisz AM, Sharkey KA, et al. Interleukin 1 beta-induced increase in substance $\mathrm{P}$ in rat myenteric plexus. Gastroenterology $1993 ; 105: 1754-60$. 